\title{
The Opacity Project and the Iron Project
}

\author{
ANIL K. PRADHAN \\ Department of Astronomy, The Ohio State University, \\ 174 West 18th Avenue, Columbus, $\mathrm{OH} 43210$, USA
}

Systematic and large-scale calculation of accurate plasma opacities and atomic data by the Opacity Project and the Iron Project has applications in many areas of astrophysics. Analysis of EUVE observations using monochromatic opacities of elements calculated by the Opacity Project is described. Theoretical methods and atomic calculations are discussed briefly. Recent work related to ionization balance, photoionization and recombination, and the modeling of plasmas in local thermodynamic equilibrium (LTE) and non-LTE are discussed. New calculations for the important iron ions, under the Iron Project, are also described and their relevance to the more extensive non-LTE calculations is pointed out. The comprehensive radiative and collisional data sets from the Opacity and the Iron Projects should be applicable to a large number of sources in the EUV and other wavelength regions.

\section{Introduction}

The Opacity Project (Seaton et al. 1994) is an international collaboration of atomic physicists and astrophysicists to calculate stellar opacities for a given chemical composition of astrophysically abundant elements. While the primary goal of the Opacity Project (hereafter OP) is to obtain accurate mean opacities for models of stellar interiors, it was found that the monochromatic opacities could be employed for the analysis and interpretation of emission spectra from stellar sources such as obtained by the Extreme Ultraviolet Explorer (EUVE) of objects like the hot photospheres of young white dwarfs at temperatures and densities normally prevalent in stellar envelopes under LTE conditions. While departures from LTE are to expected in certain cases, a detailed comparison of these models with observations should provide the basis for a more precise determination of non-LTE effects, which in turn require an even more extensive set of physical processes and parameters.

The bulk of the opacities calculations consists of atomic calculations using ab init. quantum mechanical methods that yield highly accurate radiative parameters, photoionization cross sections and radiative transition probabilities. Complementing the Opacity Project work on radiative processes, a new project called the Iron Project (Hummer et al. 1993; Bautista et al., in this volume) was initiated to address the collisional processes in astrophysical objects, particularly the spectra of Iron and other Iron peak elements in various ionization stages. These data will be applicable to the interpretation of emission line spectra and NLTE modeling of a variety of EUV sources such as the Interstellar medium, stellar coronae, flares, winds, active galactic nuclei, quasars, novae, and supernovae.

Below, we briefly describe the Opacity Project and the Iron Project (hereafter OP and IP respectively), followed by a discussion of some issues related to LTE and NLTE models and available atomic data sets for astrophysical plasma diagnostics.

\section{The Opacity Project}

It was noted by Simon (1982) that the then available opacities from the Los Alamos Opacities Library produced theoretical period ratios for Cepheid variables that disagreed widely with observations, and that an arbitrary increase in the metal opacities of factors 
of 2 or 3 appeared to ameliorate the problem. Simon issued what he termed "a plea for re-examination of the metal opacities." Two independent groups responded to the "plea": one at the Lawrence Livermore National Laboratory (Rogers \& Iglesias 1992) and the other, an international group of about 30 atomic and astrophysicists called the International Opacity Project (OP). Both groups realized that the atomic physics of the Los Alamos opacities was inadequate and incomplete, and far more radiative data was needed to account for the missing opacity. The methods involved, both in terms of the atomic physics and the equations of state, employed by the two groups were quite different. None the less, at the end of the day (actually a decade: 1983-1994) both groups obtained essentially similar opacities over most of the temperature-density regimes of practical interest. The enhancement in metal opacities in both calculations ranged from factors of 2 to 5 over the Los Alamos opacities, particularly dominated by Iron and its many ions that contributed millions of lines and continua to the overall opacity of stellar interiors. A comparison of the two sets, as well as a complete description of the OP work, is given by Seaton et al. (1994).

The quantity of particular interest in stellar models is the Rosseland mean opacity defined by

$$
\frac{1}{\kappa_{R}}=\frac{\int_{0}^{\infty} \frac{1}{\kappa_{\nu}} g(u) d u}{\int_{0}^{\infty} g(u) d u},
$$

where $\mathrm{u}=\mathrm{h} \nu / \mathrm{kT}$ and $\mathrm{g}(\mathrm{u})$ is the weighting function corrected for stimulated emission

$$
g(u)=u^{4} e^{-u}\left(1-e^{-u}\right)^{-2} .
$$

The Rosseland mean is a difficult quantity to calculate as it involves the reciprocal of the monochromatic opacity which in general is a very complicated function of the photon frequency, involving a large number of bound-bound, bound-free, free-free, and scattering contributions from all constituent atomic species in the plasma. For many complex atomic systems the opacity contribution may be a very large over a wide wavelength range. As an example, Fig. 1 shows the monochromatic opacity spectrum due to one such ion, Fe II (Nahar \& Pradhan 1994).

The OP opacities may be computed for an arbitrary mixture of elements from the monochromatic opacities of elements calculated at a wide range of temperature and density, and at a fine mesh of up to $10^{5}$ photon frequencies (with the resolution shown in Fig. 1).

The monochromatic opacities for all elements with nuclear charge $Z=1-14,16,18$, 20 , and 26 , in all ionization stages, are computed and pre-tabulated to enable the computation of mixture opacities for any chemical composition.

\section{EUVE Observations and Modeling}

The observations of hot, young white dwarfs, and their relation to stellar evolution, is one of the most important findings of the $E U V E$ program (Vennes 1995 , in this volume). The analysis of spectra of white dwarfs early in their evolution is essential to the determination of the precise evolutionary path, along the H-R diagram, from the planetary nebula phase down to the stable, final state. As the ejecta from the PN phase is driven by radiation pressure on the one hand, and the gravitational stratification of elements in the remnant core takes place on the other hand, some interesting "inversions" in the photospheres are observed. Iron, owing to the rich and complex atomic structure of its low ionisation stages and its relatively high abundance, is radiatively levitated by the opacity inherent in the millions of possible transitions, primarily in the UV and the EUV. 


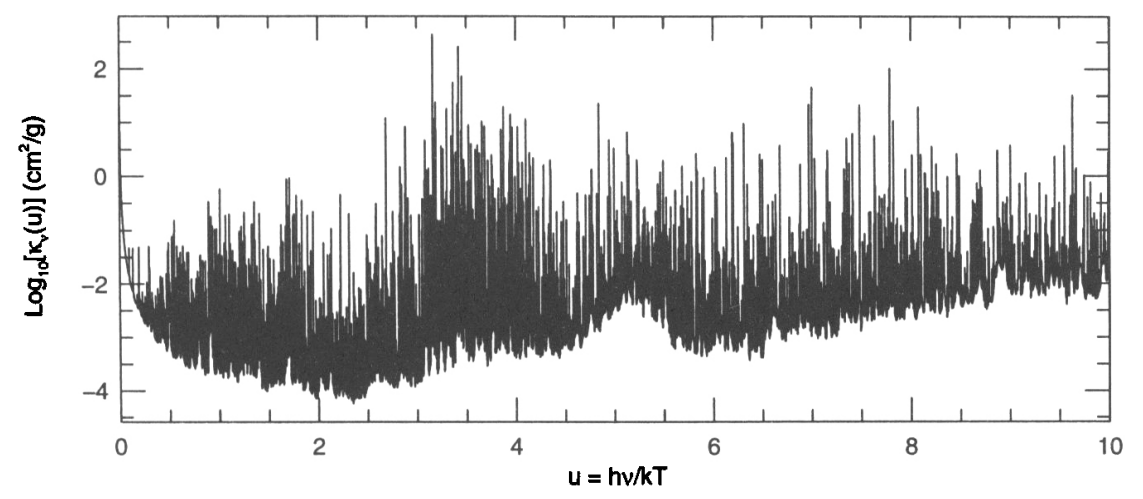

FIgURE 1. Monochromatic opacity of Fe II at $\log T=4.2, \log N_{e}=16$.

For example, the $E U V E$ observations reveal the EUV spectra to be dominated by Iron in certain wavelength regions. A full-scale theoretical computation therefore depends on an accurate and complete determination of the opacities of constituent elements.

The emissivities of the elements in the emitting photospheres can be derived in LTE. The medium resolution spectra can be modeled using the emissivities of elements in the emitting photospheres calculated in LTE from the monochromatic opacities of elements, with abundances optimised to fit the spectrum - thus determining the composition of the white dwarf photosphere. The observations clearly show the prominent contribution of iron which is radiatively excited in the intense radiation field and emits copiously in the EUV, in particular through the ionization stages Fe IV and Fe V. Several other elements have also been identified. It is necessary to calculate the monochromatic opacities of all of these elements at a T- $\rho$ mesh appropriate to the photospheres of hot white dwarfs: $\log T=4.5-5.5, \log N_{e}=12-20$.

The calculated opacities include all the main atomic processes: bound-bound, boundfree, free-free, electron scattering, and line broadening.

The presence of elements in the white dwarf photospheres may be deduced from spectral observations and certain spectral features. A number of elements have been observed in the $I U E$ and the EUVE spectra of white dwarfs (Vennes, this volume). Among these are $\mathrm{C}, \mathrm{N}, \mathrm{O}, \mathrm{Si}, \mathrm{S}$, and $\mathrm{Fe}$. In Fig. 2 we present the theoretical emissivities, calculated using the monochromatic opacities, as each new element is added to the mixture. The contribution of the metal opacities is clearly seen. While the contribution of silicon and sulfur is small, and manifests only certain detailed spectral features, the dramatic effect of the iron opacity on the entire background in the lower wavelength region is evident. In order to emphasize the contribution of the individual opacities, the computed fluxes in Fig. 2 do not include the effect of the interstellar medium and therefore may not be directly compared to the observations (the spectra have been smoothed to a resolution of $1 \AA$ to match the resolution of the $E U V E)$.

A representative model of the theoretically computed flux is compared with the EUVE observations of a young white dwarf in Fig. 3. Work is in progress to improve the models by more accurate estimates of abundances, the inclusion of other observed elements such as neon and magnesium, and departures from NLTE (discussed below). In particular the discrepancies between observations and theory may be attributable to the fact that the current $\mathrm{OP}$ data for Fe I-V is not very accurate, and is being recalculated to higher ac- 


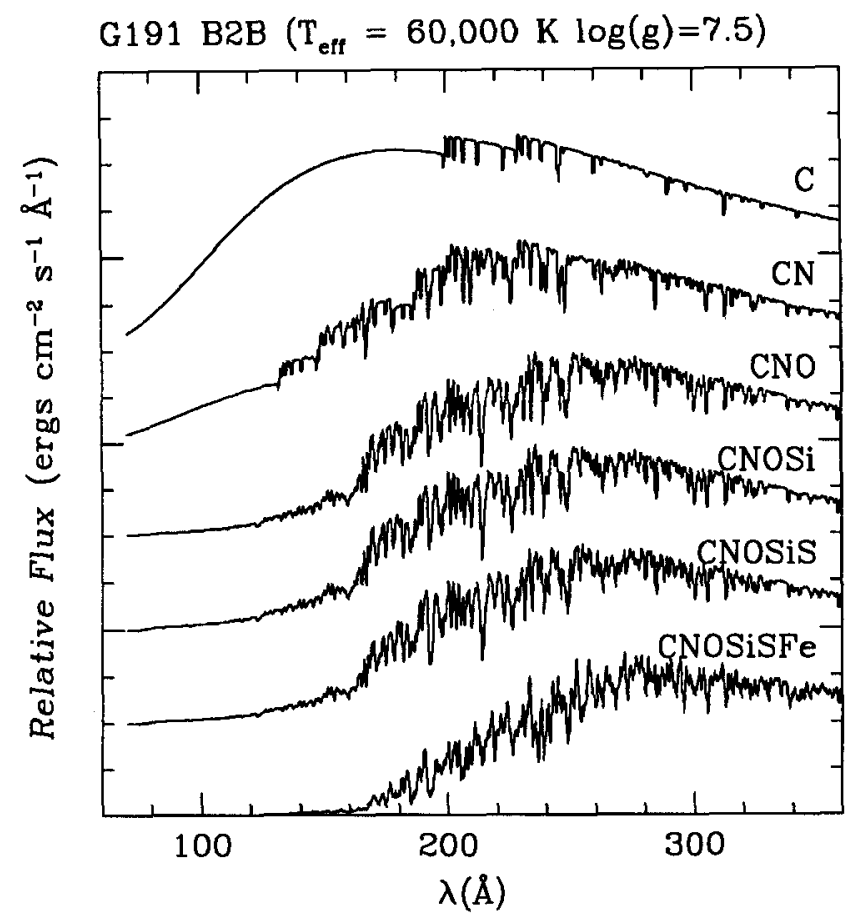

Figure 2. Incremental mixture emissivities of $\mathrm{C}, \mathrm{N}, \mathrm{O}, \mathrm{Si}, \mathrm{S}$, and Fe. The bottom-most curve demonstrates the dominant effect of iron on the overall, as well as the detailed, opacity of the plasma. All abundance fractions relative to $\mathrm{H}$ are $3 \times 10^{-6}$.

curacy under the IP (Nahar \& Pradhan 1994; Bautista \& Pradhan 1995a). For example, work in progress for $\mathrm{Fe} \mathrm{V}$ shows that the new data will yield significantly higher opacity in the wavelength range $<300 \AA$.

\section{Non-LTE Models: Collisional and Recombination Data}

While departures from LTE would not be unexpected, the precise nature of such effects is difficult to determine quantitatively since extensive NLTE calculations are necessary. Such calculations need to include all relevant radiative and collisional processes important in line formation, coupled with an accurate treatment of radiative transfer (e.g., Hubeny \& Lanz 1995). A huge amount of accurate atomic data is needed. We describe below two projects that are extensions of the Opacity Project and are devoted to large-scale calculation of collisional and recombination parameters required in NLTE codes.

\subsection{The Iron Project}

The primary aim of the Iron Project is to compute collisional data for the iron-peak elements in various ionization stages (Hummer et al. 1993). The first two phases of the project deal with fine structure transitions along several isoelectronic sequences and for all iron ions. Some radiative data for low ionization stages of iron ions, Fe I-V, is also being computed to higher accuracy than in the earlier work from the Opacity Project 


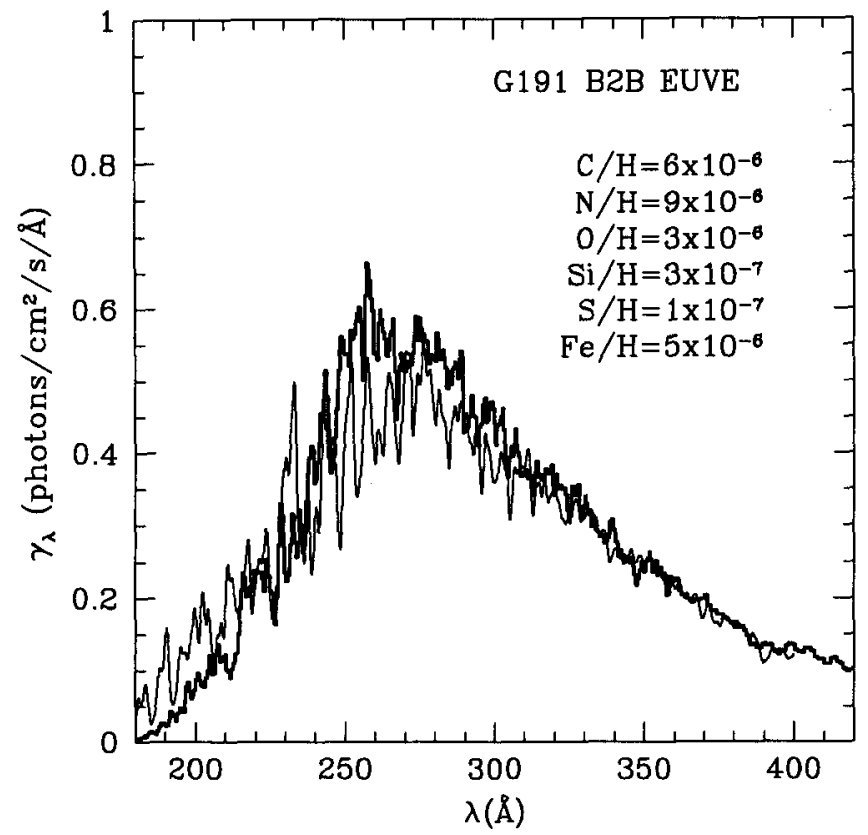

FIgure 3. Comparison of the theoretical emissivity (thin line) and the observed $E U V E$ spectra (thick line). The white dwarf model assumes the given chemical abundance ratios, at $T=56,000$ $\mathrm{K}$ and $\log g=7.5$.

(e.g., Nahar \& Pradhan 1994). Information on the IP and the latest list of publications may be obtained on World Wide Web (URL: http://www.am.qub.ac.uk/projects/iron/).

The IP work has applications in both the LTE and the NLTE modeling of EUVE spectra. The new radiative calculations for $\mathrm{Fe} I$ and $\mathrm{Fe} I I$, and the opacities calculations therefrom, differ greatly from the earlier OP data. For example, Fig. 4 shows the recently calculated photoionization cross section for the ground state of Fe I, which is up to 3 orders of magnitude higher than the data currently available (Bautista \& Pradhan 1995a).

The NLTE models require collisional cross sections and rates for a large number of transitions in many of the iron ions. Recently Zhang \& Pradhan $(1995 a, b)$ have calculated electron impact excitation rate coefficients for $10,011 \mathrm{IR}, \mathrm{O}$, and UV transitions in Fe II and 23,871 transitions in Fe III. Similar calculations are in progress for Fe IV, Fe V and Fe VI. New effective collision strengths for Ni II have also been computed recently (Bautista \& Pradhan 1995b).

Further details on the IP work are given in the papers in this volume by Bautista et al. and Mason; the latter work deals with the important coronal ions of iron.

\subsection{Unified and Consistent Electron-Ion Recombination Rates}

Electron-ion recombination calculations, consistent with the Opacity Project photoionization data, are being carried out to determine, for the first time, accurate and selfconsistent ionization balance in astrophysical sources, as well as for NLTE populations dependent on partial photoionization and recombination cross sections for individual levels. 


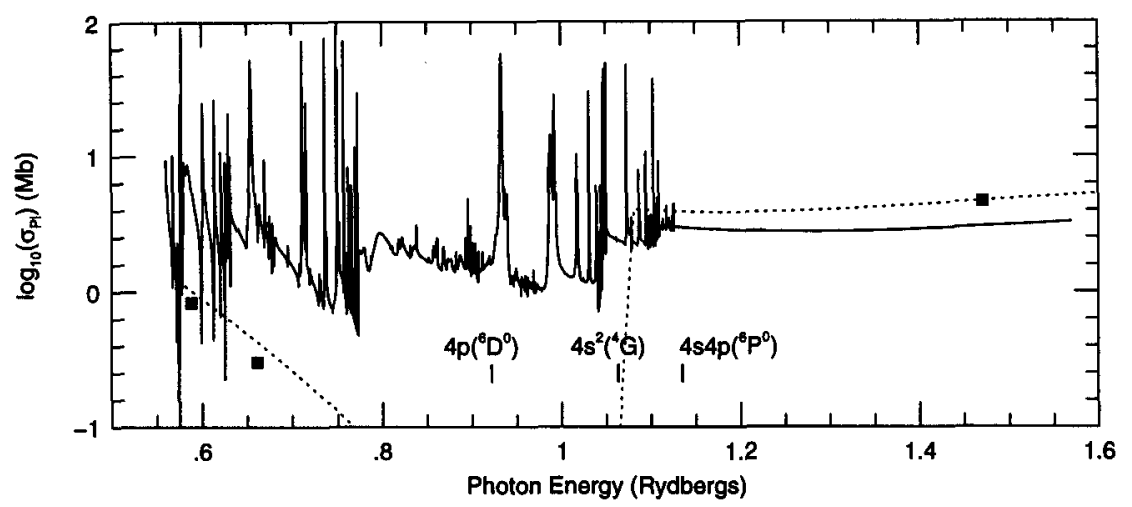

FIGURE 4. Photoionization cross section of the ground state $3 d^{6} 4 s^{2}\left({ }^{5} D\right)$ of Fe I; full curve = Bautista \& Pradhan (1995a); filled squares = Reilman \& Manson (1979); broken curve = Verner et al. (1993). The orders of magnitude enhancement over the previous calculations is primarily due to the coupling among various states dominated by the open $3 d$ and the $4 s$ subshells, which is also manifest in the autoionization resonances.

Accurate Recombination rates are crucial to the calculation of ionization balance in plasmas; however an equally important point is to obtain both the recombination and the ionization cross sections rates within the same formulation in order to be self-consistent. A new method has recently developed by Nahar \& Pradhan (1995, and references therein) to calculate unified electron-ion recombination rates that include both the radiative and the di-electronic recombination ( $R R$ and $D R$ ) processes in an ab init. manner, giving a single, total recombination rate coefficient $\alpha_{R}(T)$ for each atom or ion as a function of temperature. These recombination rates are calculated using the same atomic physics (i.e., eigenfunction expansions for the electron+ion system) as in the OP work, and therefore are fully consistent with the new OP photoionization cross sections (which may be obtained from TOPBASE, Cunto et al. 1993). It might be emphasized that the currently available $R R$ and $D R$ rates are calculated in different approximations and are, in general, inconsistent with the photoionization cross sections, possibly leading to considerable loss of accuracy in ionization balance calculations.

Fig. 5 illustrates the unified recombination rate coefficients obtained with the new method for an isoelectronic sequence of ions (carbon-like). Along the sequence the $\alpha_{R}(T)$ increase with nuclear charge $Z$ in the low temperature region, where the background recombination (i.e., RR type) dominates, but the relative importance of DR diminishes with $\mathrm{Z}$ converging to a wide bump in the figure in the high- $T$ region ( $\log T>5.0$ ). In the low- $T$ region the resonant recombination (DR type) may also contribute for some ions, as indicated by a smaller bump.

\subsubsection{Photoionization and Recombination into Individual States}

In addition to the total photoionization and recombination rates, it is also necessary to compute partial photoionization and recombination rates for the individual levels of an ion populated in NLTE. Whereas some such calculations are in progress, these are extremely difficult for the complex iron ions where the levels of each ion are coupled to the levels of adjacent ions through photoionization and recombination. The calculations under way should yield the partial photoionization and recombination rates for the metastable levels of the ions concerned; the partial rates being dependent upon the 


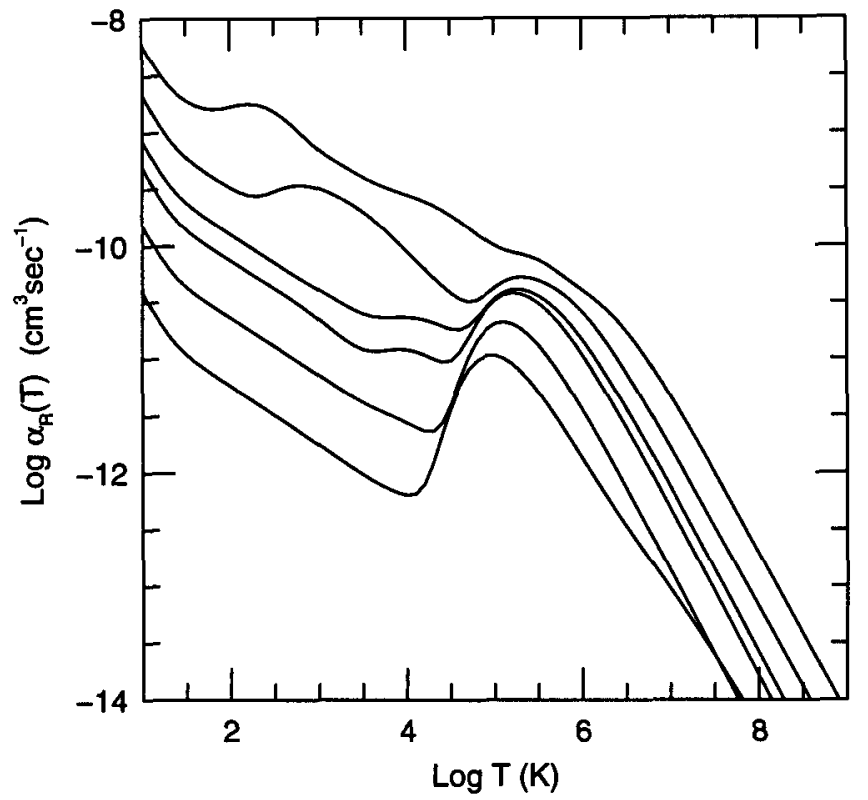

FIGURE 5. Total electron-ion recombination rate coefficients for ions in the carbon sequence; the curves represent recombination rates for C I, N II, O III, F IV, Ne V, and Si IX from bottom to top (Nahar \& Pradhan 1995). Applicable to ionization balance calculations, these unified rates include both the radiative and the di-electronic recombination processes and are consistent with the OP photoionization data.

individual level populations which, in turn, may be determined from the solution of a collisional-radiative model. For Fe I-V, for example, a number of such metastable levels need to be considered, dominated by the lowest even parity configurations of the type $3 d^{n} 4 s^{m}$ which dominate the wavefunctions of a relatively large number of terms. Given the large differences in the photoionization cross sections from available data for these ions (e.g. Fig. 4), it is clear that current photoionization models need considerable revision in order to incorporate the new photoionization and recombination data.

\section{Conclusion}

The Opacity Project and the Iron Project have enabled a far more extensive and detailed treatment of radiative and collisional processes in astrophysical models than heretofore possible. The huge amount of atomic data computed by the Opacity Project and the Iron Project should meet most of the needs of the next generation of LTE and NLTE models. Information on databases and ongoing work on the OP and the IP may be obtained from the author (pradhan@payne.mps.ohio-state.edu).

I would like to thank Stephane Vennes, Sultana Nahar, and Manuel Bautista for some of the figures. This work is partially supported by the US National Science Foundation (PHY-9421898), the NASA LTSA program (NAGW-3315), and the NASA ADP program (NAS 5-32643). The computational work is carried out on the CRAY Y-MP8/64 and the MPP CRAY T3D at the Ohio Supercomputer Center in Columbus, Ohio. 


\section{REFERENCES}

Bautista, M. A. \& Pradhan, A. K. 1995a, Photoionization of neutral Iron, J. Phys. B Lett., in press

Bautista, M. A. \& Pradhan, A. K. 1995b, Atomic Data from the Iron Project XIV. Electron excitation rates and emissivity ratios for forbidden transition in Ni II and Fe II, A\&A, submitted

Cunto, W., Mendoza, C., Ochsenbein, F., \& ZeIPPen, C. J. 1993, TOPbase at the CDS, A\&A, 275, L5

Hummer. D. G., Berrington, K. A., Eissner, W., Pradhan, A. K., Saraph, H. E., \& Tully, J. A. 1993, Atomic data from the IRON Project, A\&A, 279, 298

Nahar, S. N. \& Pradhan, A. K. 1994, Atomic data for opacity calculations: XX. Photoionization cross sections and oscillator strengths for Fe II, J. Phys. B: At. Opt. Phys., 27, 429

Nahar, S. N. \& Pradhan, A. K. 1995, Unified electron-ion recombination rate coefficients for Sulfur and Silicon Ions, ApJ, in press

ReLman, R. F. \& Manson, S. T. 1979, Photoabsorption cross section for positive atomic ions with $\mathrm{Z} \leq 30$, AJSS, 40,815

Rogers, F. J. \& Iglesias, C. A. 1993, Radiative atomic Rosseland mean opacity tables, ApJS, 79, 507

Seaton, M. J., Yu, Y., Mrhalas, D., \& A. K., Pradhan 1994, Opacities for stellar envelopes, MNRAS, 266, 805

SimON, N. R. 1982, A plea for re-examination of metal opacities, ApJ, 260, L87

Verner, D. A., Yakovlev, D. G., Band, I. M., \& Trzhaskovskaya, M. B. 1993, Subshell photoionization cross sections and ionization energies of atoms and ions from $\mathrm{He}$ to $\mathrm{Zn}$, At. Data Nucl. Data Tables, 55, 233

Zhang, H. L. \& Pradhan, A. K. 1995a, Atomic Data from the Iron Project VI. Collision strengths and rate coefficients for Fe II, A\&A, 293, 953

Zhang, H. L. \& Pradhan, A. K. 1995b, Relativistic and electron correlation effects in electron impact excitation of $\mathrm{Fe}^{2+}$, J. Phys. B: At. Opt. Phys., submitted 